
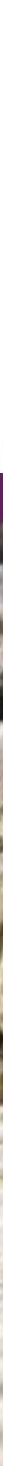

Référence complète de l'article:

Coenga-Oliveira, D. et Anctil Avoine, P. (2017). "Conformez-vous!»: Les résistances et contestations à la marchandisation du savoir dans l'université néoliérale. REVISTA TEMAS, 3(11), 13 - 27.

\title{
«Conformez-vous!»: Les résistances et contestations à la marchandisation du savoir dans l'université néolibérale ${ }^{1}$
}

\author{
Danielle Coenga-Oliveira ${ }^{2}$ \\ Priscyll Anctil Avoine ${ }^{3}$
}

Recibido 23/06/2017 Aprobado 15/07/2017

\section{Résumé}

Le présent article est le résultat des réflexions et débats qui se sont générés à propos de l'université contemporaine lors du Séminaire doctoral en science politique à l'Université du Québec à Montréal. Les changements économiques et politiques des années 1970 ont impulsé une réorganisation du processus de production de la connaissance. Afin de s'adapter au nouveau contexte sociopolitique de libéralisation des marchés et des efforts des États pour établir la compétitivité des économies nationales, les universités ont dû subir une grande restructuration. L'université néolibérale est alors caractérisée par une exigence de productivité accrue, par des compressions budgétaires, par l'accumulation de tâches administratives pour les enseignant-e-s ainsi que par une marchandisation des savoirs. En ce sens, cet article vise à s'interroger sur la néolibéralisation de l'université contemporaine et sur les transformations pratiques qui la caractérisent dans le but de proposer des pistes de réflexion sur les résistances possibles à la précarisation de l'institution par le système néolibéral. II présente un bref contexte sur l'émergence de l'université néolibérale et les caractéristiques de ce type d'université. Dans un deuxième temps, l'article aborde des pistes de résistance à la marchandisation de la connaissance et, finalement, il propose une réflexion sur la contestation des savoirs hégémoniques. En bref, I'article invite à la remise en question de la logique de productivité qui sous-tend l'université néolibérale et cache un système d'homogénéisation de la pensée qui contribue à la construction des savoirs hégémoniques aboutissant à la marginalisation des savoirs subalternes.

\section{Mots-clés}

Université néolibérale, savoirs hégémoniques, capitalisme cognitif, marchandisation du savoir, savoirs subalternes.

1. Article de réflexion qui reprend les débats et papiers discutés lors du Séminaire de préparation à la rédaction de textes scientifiques et aux activités de recherche sous la direction de Carole Clavier à l'Université du Québec à Montréal. Nous remercions Carole Clavier ainsi que nos collègues qui nous ont permis de repousser constamment les limites de nos acquis.

2. Psychologue, Maîtrise en Psychologie sociale de l'Université de Brasilia (Brésil), Maîtrise en Études internationales de l'Université de Montréal (Canada) et doctorante en Science politique et études féministes à l'Université du Québec à Montréal. Courriel: coenga_oliveira.danielle@ courrier.uqam.ca

3. Professionnelle en Études internationales et langues modernes de l'Université Laval (Canada), Maîtrise en Paix, conflits et développement de I'Universitat Jaume I (Espagne) et doctorante en Science politique et études féministes à l'Université du Québec à Montréal. Boursière Vanier Banting 2017-2020. Courriel : anctil_avoine.priscyll@uqam.ca 


\section{“iConfórmense!” Resistencias y contestaciones a la mercantilización del conocimiento en la universidad neoliberal}

\section{Resumen}

Este artículo presenta los resultados de las reflexiones llevadas a cabo en el Seminario de Doctorado en Ciencias Políticas en la Université du Québec à Montréal (Canadá), a propósito de la universidad contemporánea. Los cambios económicos y políticos de la década de 1970 provocaron una reorganización del proceso de producción de conocimiento. Para adaptarse al nuevo contexto socio-político de la liberalización del mercado y frente a los esfuerzos de los Estados para adaptar la economía nacional a la competitividad, las universidades han tenido que someterse a una importante reestructuración. Así, podemos decir que la universidad neoliberal se caracteriza por altas exigencias en materia de competitividad, recortes en los presupuestos, acumulación de tareas administrativas para el cuerpo docente, y por una mercantilización del conocimiento. En este sentido, este artículo tiene como objetivo analizar el proceso de neoliberalización de la universidad contemporánea y las transformaciones prácticas que la caracterizan, con el fin de proporcionar elementos de reflexión sobre las posibles formas de resistencia a la precariedad institucional frente al sistema neoliberal. El escrito aporta un breve contexto sobre la emergencia de la universidad neoliberal y, luego, presenta las características de este tipo de universidad. En tercer lugar, discute las oportunidades de resistencia frente a la comercialización del conocimiento y, por último, se propone una reflexión sobre la contestación de los saberes hegemónicos. En resumen, el artículo invita a cuestionar la lógica de productividad que sustenta la universidad neoliberal, la cual esconde una homogeneización del sistema de pensamiento que contribuye a la construcción de un saber hegemónico y a la marginalización de los conocimientos subalternos.

\section{Palabras clave}

Universidad neoliberal, saberes hegemónicos, capitalismo cognitivo, mercantilización del saber, saberes subalternos.

\section{Introduction}

L'université est l'endroit de production de la pensée critique par la discussion approfondie de sujets complexes. Elle permet aux individus qui y sont de vivre une expérience unique d'enrichissement intellectuel et d'épanouissement culturel. Cette conception de l'université est encore bien répandue dans l'imaginaire social, mais qu'en est-il vraiment de cette vision idéalisée? Bien que cette représentation de l'université soit acceptée et défendue par la plupart des étudiant-e-s, chercheur-e-s et professeure-s, elle est de plus en plus remise en doute. En effet, le modèle d'université, comme une institution autonome de production du savoir qui permettrait la formation des sujets critiques capables de promouvoir l'avancement des sciences et de chercher à résoudre des problèmes d'ordre social, est en crise.

En partant de ce constat, il apparaît nécessaire de repenser le modèle de construction de la connaissance priorisé dans les universités contemporaines. Ainsi, notre intention dans cet article est de nous interroger sur la néolibéralisation ${ }^{4}$ de I'université contemporaine et sur les transformations pratiques qui la caractérisent dans le but de proposer des pistes de réflexion sur les résistances possibles au système néolibéral. Pour se faire, l'argumentaire est divisé de la façon suivante : premièrement, nous abordons la méthodologie et puis, nous exposons un bref contexte sur l'émergence de

4. Suivant la proposition de Mountz et al. (2015), nous adoptons le concept de néolibéralisme proposé par Sparke $(2006, \mathrm{p} .153)$ comme " a contextually contingent articulation of free market governmental practices with varied and often quite illiberal forms of social and political rule ". 
I'université néolibérale. Nous poursuivons avec les caractéristiques de ce type d'université avant d'aborder certaines pistes pour fomenter un débat sur les formes de résistance à la néolibéralisation de la connaissance dans les universités contemporaines. Finalement, nous proposerons une réflexion sur la contestation des savoirs hégémoniques.

\section{Méthodologie}

Le présent article est le produit d'une réflexion continue durant la session d'hiver 2017 pendant le Séminaire de préparation à la rédaction de textes scientifiques et aux activités de recherche sous la direction de Carole Clavier à I'Université du Québec à Montréal. II dérive des lectures et débats qui se sont donnés lors des séances qui avaient un double objectif: (1) "produire une réflexion théorique sur les enjeux de la production et de la mobilisation de la recherche à partir des lectures identifiées dans le plan de cours" et (2) «se préparer à la production et à la diffusion de la recherche par différents exercices pratiques de rédaction et de communication des connaissances tout au long de la session" (Clavier et D'Aoust, 2017).

Ainsi, la méthodologie s'est divisée de la manière suivante: certains textes étaient attitrés à chaque séance de cours sur une période de 15 semaines dans le but de provoquer des réflexions critiques autour de l'université néolibérale et du complexe panorama que les doctorante-s contemporain-e-s confrontent. Puis, chaque semaine, les étudiant-e-s étaient amené-e-s à consolider des réponses argumentatives sur les textes analysés et postérieurement discutés dans le cadre du cours. Le présent texte est le produit du dialogue entre deux doctorantes sur les réflexions issues de ce séminaire.

\section{Contexte: capitalisme cognitif et économie du savoir}

El capitalismo sabe dosificar la violencia que necesita para mantenerse.

[Le capitalisme sait doser la violence qu'il nécessite pour se maintenir] Gearóid Ó Loingsigh

Les changements économiques et politiques des années 1970 ont impulsé une réorganisation du processus de production de la connaissance. Afin de siadapter au nouveau contexte sociopolitique de libéralisation des marchés et des efforts des États pour établir la compétitivité des économies nationales, les universités ont dû subir une grande restructuration (Lesemann, 2003; Insel, 2009). En tant qu'institution centrale de recherche et d'enseignement, I'université est un acteur social clé dans le processus de changements sociaux et économiques. Sa fonction qui était auparavant de construire la connaissance et d'établir les fondements pour une recherche intellectuelle (Côté et Allahar, 2010) devient maintenant celle de produire des savoirs dans les domaines d'intérêts des gouvernements et des organisations privées pour atteindre l'objectif de rétablir une économie forte en constante croissance (Lesemann, 2003). C'est dans ce contexte que s'inaugure une nouvelle forme du capitalisme, soit le "capitalisme cognitif» et qui fait "de la connaissance le lieu central de l'accumulation du capital»" (Insel, 2009, p.142-143).

Dans cette nouvelle économie du savoir, pour reprendre les mots d'Insel (2009), la connaissance qui était conçue comme un bien immatériel est devenue une marchandise. Cette transformation a été possible grâce à un complexe jeu de forces qui agissent dans le rapport entre les acteurs et les institutions sociales divers - comme l'université, 
le gouvernement, les intellectuel-le-s et les organismes de subvention. En s'appuyant sur des discours voulant que la croissance économique soit le résultat de l'innovation du marché, l'État et les organismes de subvention à la recherche ont profondément dirigé la production scientifique vers leurs domaines d'intérêts - comme exemple, par l'imposition de conditionnalités aux financements des recherches. Comme le souligne Lesemann (2003), dans ce nouveau modèle de gouvernance de la production universitaire, l'État, les organismes de subvention, aussi bien que les grandes institutions internationales (telles que I'UNESCO, I'OCDE et la Banque Mondiale), adhèrent à une conception utilitariste de la recherche. Dans ce sens, guidée par des intérêts de croissance économique, la production scientifique rentre dans une logique de productivité qui travaille en fonction des intérêts nationaux.

Dès lors, le prestige, la reconnaissance et le pouvoir de l'université en tant qu'institution reconnue par sa grande valeur sociale et par son rôle de formation critique des individus, perd du terrain face à lıutilisation de la construction du savoir à des intérêts particuliers de marché. Selon Olivier (2015) et Meulemeester (2011), les nouvelles exigences des organismes de subvention de recherche, les intérêts politiques des gouvernements, les exigences d'augmentation des places dans les universités et l'intérêt remarquable dans le diplôme comme outil de faire accroître les salaires des étudiantes seraient des facteurs d'explication de la manière actuelle de l'université répondre aux intérêts néolibéraux.
En ce sens, il est possible d'affirmer que I'université comme institution sociale, culturelle et politique oscille entre des changements, issus du système économique néolibéral et de "l'économie du savoir", et des continuités réaffirmant les héritages euro-centrés et millénaires de cette institution (Gingras, 2004). Cette ambivalence entre céder aux standards mercantiles actuels et de perpétuer d'un système ancien où les universités, financées par l'État, était protégées des pressions et des exigences de productivité et de court terme (Meulemeester, 2011) fait que l'institution ne répond pas à une fonction sociale claire. Elle est devenue " the new everything " comme l'argumente Considine (2006, p.258) et la pression sur la performance des scientifiques est de plus en plus forte. Le modèle et la motivation de la production de la connaissance académique deviennent ceux des entreprises : le désir de l'argent et l'obligation de l'obéissance (Meulemeester, 2011). Autrement dit, il fallait des changements pour qu'elle garantisse sa survie dans un contexte social et économique de plus en plus compétitif, et que de moins en moins la permet d'être autonome.

Considérant que "la capacité d'adaptation" est une caractéristique remarquable de la force millénaire de I'université, comme l'affirme Yves Gingras (2004), il est important de souligner que, de nous jours, elle peine d'une part à repenser ses propres cadres et aussi qu'elle est entrée dans une logique de "quasimarchés" (Meulemeester, 2011, p.14) qui nuit à ses principales missions - celles de la recherche et de l'enseignement. En ce sens, la néolibéralisation de l'université contribue à la précarisation de la qualité de l'enseignement et de la recherche et des conditions d'emploi et d'étude dans I'institution. 


\section{La marchandisation de du savoir et l'impact sur le travail de recherche et d'enseignement}

"Quelle est votre valeur sur le marché scientifique?" : telle est la question posée par Chamayou (2009, p.208) visant à montrer les comparaisons avérées entre les rouages des marchés financiers et les structures de compétition du système éducatif. En effet, nos statistiques Google Scholar et notre Indice $H$ sont les marques de notre valeur et les processus d'accréditation institutionnels le savent: dans le contexte de l'économie du savoir et du capitalisme cognitif, nous assistons à la " marchandisation du savoir» (Insel, 2009, p.142) et donc, à la quantification d'un bien jugé jusqu'à maintenant immatériel, la connaissance. Les chercheur-e-s se confrontent de plus en plus à des standards de productivité difficiles à atteindre, avec le développement de plusieurs indicateurs de production scientifique plutôt questionnables. C'est ainsi que les juges de la société du savoir apparaissent comme des organisations commerciales privées s'occupant d'encadrer le "marché de la connaissance» (Insel, 2009 p.146) et de classer les équipes de recherche selon leur performance. Sous le prétexte de fournir des données qui permettraient une comparaison juste entre les chercheurs et les universités, en établissant un classement des chercheure-s, ces sociétés privées dictent les règles d'évaluation de la productivité académique de partout dans le monde. Alors, dans un contexte où le classement des professeurs et des universités a un impact direct sur la réussite de subventions à la recherche, ces organisations ont un pouvoir immensurable sur la production de la connaissance et les transformations des modes de production scientifique. Cette section fait l'analyse de ces transformations dans les universités contemporaines en abordant les problématiques suivantes: la compétition entre les pairs, la mécanisation du travail intellectuel ainsi que la course aux subventions et aux publications.

Dans un premier temps, nous estimons que la néolibéralisation des milieux scientifiques, par sa logique marchande, conduit inévitablement à la compétition accrue entre les pairs, ce qui amène aussi les doctorant-e-s et professeur-e-s à dévaloriser certaines formes de travail et à remplacer la créativité intellectuelle par la performance pour éviter la précarisation socioéconomique. La compétitivité se fait ressentir de manière prononcée au doctorat: Cornut et Larivière (2012) ainsi que Koromyslova et Depelteau (2015) associent les abandons au niveau doctoral surtout par le manque ou l'absence de financement, qui devient un véritable casse-têtes pour les jeunes chercheur-e-s. Plus encore, il semblerait que les difficultés de financement dû à une exigence de performance toujours plus élevée soit aussi une source de compétitivité entre les doctorant-e-s, qui tentent de faire leur place dans cette course aux bourses, symptôme d'une économie intellectuelle basée sur les principes issus de la doctrine du néolibéralisme.

Au Canada et en Amérique en général, l'université comme institution est largement marquée par l'effet homogénéisant de la recherche étatsunienne sur les disciplines (Tupper et Pocklington, 2002). Et donc, encore une fois, certain-e-s chercheur-e-s, certaine-s étudiant-e-s, voire certains pays, sont hautement marginalisé-e-s de cet épicentre ce qui diminue l'accès à l'emploi, ajoutant une couche de compétitivité, celle de la langue. L'enjeu n'est pas de s'ouvrir sur l'apprentissage d'une deuxième langue, mais d'apprendre la langue qui permet l'ascension professionnelle, 
même si cela implique la dislocation des structures de pensée. Selon Grondin, D'Aoust et Racine-Sibulka, la langue anglaise, considérée comme langue internationale, devient donc un capital culturel. Elle est source de crédibilité et permet aux anglophones de publier dans les revues les plus prestigieuses (à savoir, possédantes d'un important facteur d'impact) et d'avoir une plus grande employabilite que les non-anglophones. Ainsi, comme l'affirment les auteur-e-s, " la décision pour un étudiant de poursuivre un doctorat en français au Canada est lourde de conséquences " $(2012$, p.22) du point de vue de la compétitivité.

En conséquence, la compétitivité entre les pairs se fait sentir de plus en plus, augmentant le même sentiment de précarité et d'isolement qui semble insinuer que la réussite des chercheur-e-s dépend surtout de leur performance par rapport aux autres. Les étudiant-e-s se retrouvent en état de permanente compétition avec leurs pairs en plus de devoir montrer leurs capacités de leadership aux selon les nouveaux paramètres des organismes subventionnaires: un "profil parfait auquel personne ne correspond réellement» (Koromyslova et Depelteau 2015, p.265). Finalement, comme Grondin, D'Aoust et Racine-Sibulka (2012) le suggèrent, la compétition se manifeste par les faibles perspectives d'emploi dans le milieu universitaire ce qui amène les étudiant-e-s à devoir publier et faire des conférences de manière plus soutenue et fréquente pour atteindre les exigences d'excellence du système néolibéral.

En deuxième lieu, nous considérons que I'ère universitaire néolibérale a provoqué une certaine désintellectualisation du travail scientifique, considérant que le modèle économique rend plutôt machinal les actions quotidiennes des professeure-s, chercheur-e-s et étudiant-e-s : «we ignore the various institutional stupidities that we reproduce on a daily basis " (Paterson, 2015, p.223). C'est une logique bureaucratique plutôt qu'intellectuelle, qui tente de répondre de plus en plus aux impératifs du capitalisme cognitif. En nous référant à nos propres expériences professionnelles, nous pouvons observer que nous sommes entrées dans une logique de compter sans cesse le temps perdu ou gagné alors qu'une panoplie de système de gestion académique sont implantés pour contrôler les tâches de chaque personne au sein de l'institution, ce que Mountz et al. (2015, p.1240) appellent des "digital measures». Nous sommes entré-e-s- à l'ère de la «chaîne de production du savoir» (Insel, 2009, p.145), dont le but est de nous faire comprendre que notre autonomie face au système est très réduite: à l'image de l'industrie, nous devons maintenant justifier notre temps par sa rentabilité et démontrer que nos activités de recherche et administratives donnent un bénéfice matériel à l'université. $C^{\prime}$ est ainsi que les scientifiques passent des heures à les justifier: en moins de temps, il faut faire plus; plus de rapports, plus de publications, plus de travaux à corriger. Comme le soulignent certaines auteures féministes, ce sont les "everincreasing demands of academic life: the acceleration of time in which we are expected to do more and more" (Mountz et al., 2015, p.1237). Et donc, nous nous retrouvons maintenant à nous parler en termes néolibéraux avec les indicateurs, la production scientifique, l'indice $\mathrm{H}$, I'innovation technoscientifique; nous finissons par oublier pourquoi nous faisons partie d'une communauté scientifique, c'est-à-dire, pour la pédagogie et la construction - et non la production - des savoirs. Nous sommes devenu-e-s " growth-maniacs» (Paterson, 2015, p.327).

En troisième lieu, cette obsession pour la croissance de l'université néolibérale 
a pour effet de changer drastiquement les conditions de financement (public et privé) et de publications scientifiques. $C^{\prime}$ est de cette manière que la quête du savoir et de la vérité s'éclipsent puisque les professeur-e-s et chercheur-e-s doivent employer tous leurs efforts pour réussir à décrocher des subventions de recherche, à publier dans une revue scientifique reconnue et à présenter leurs travaux dans les conférences un peu partout dans le monde pour tenter de bâtir un capital symbolique (Grondin, D'Aoust et Racine-Sibulka, 2012; Bourdieu, 1979).

Selon Bartkowski, Deem et Ellison, les instances d'évaluation ou "departamental evaluation and institutional accreditation process" (2015, p.101) fomentent une culture de l'évaluation qui met l'accent sur la production à la chaîne d'écrits dits "scientifiques" ce qui garantit souvent I'accès aux postes permanents et à des augmentations de salaire (Maliniak, Powers et Walter, 2013). De cette culture de l'évaluation, découle aussi les possibilités pour les subventions de recherche qui, à leur tour, sont indispensables pour le rayonnement des chercheur-e-s et l'appui aux étudiant-e-s doctorant-e-s. D'une part, dans le cadre néolibéral, les organismes publics et privés de subvention de recherche basent leurs discours de libéralisation des universités sur des arguments d'innovation à la croissance économique. Ces arguments, qui de prime abord ne semblent pas être à la défense des intérêts et des domaines spécifiques, sont intimement corrélés avec les intérêts du marché dans les champs de la santé, de l'industrie pharmaceutique et des secteurs des ressources naturelles par exemple (Lesemann, 2003). Du côté des bourses attribuées aux étudiant-e-s, le profil d'étudiante cherché par les grands organismes de subventions est celui de chercheur-e multitâches (Koromyslova et Depelteau, 2015). Ces étudiant-e-s, qui auront des avantages clairs dans leur parcours académique, par exemple plus de publications, plus de chances d'être employés au sein de l'université et besoin de moins de temps pour conclure les études (Cornut et Larivière, 2012), doivent être aussi des leaders dynamiques et autonomes pour répondre aux impératifs du marché: il faut être engagé, faire du bénévolat, posséder des expériences de travail ainsi des prix et distinctions. Et la compétition se fait de plus en plus forte puisque le système néolibéral, par définition, ne peut pas ralentir la cadence.

D'autre part, les groupes éditoriaux (comme Elsevier ou Thomson Reuters par exemple) qui dominent le marché de la publication scientifique sont ceux qui définissent les règles du jeu en la matière, créant aussi des rapports de force inégaux mondialement. Le système d'évaluation instauré par ces groupes éditoriaux s'apparente à un véritable monopole ayant des diktats bien précis:

Elsevier numéro un du secteur qui possède $16 \%$ des revues scientifiques publiées dans le monde. Si vous ajoutez les autres géants que sont Springer Nature, Wiley Blackwell's et Taylor \& Francis, vous obtenez une mainmise qui grimpe à $40 \%$ du marché. (Data Gueule, 2016)

De la sorte, il semble que l'activité des scientifiques soit hautement reliée à la possibilité pour les chercheur-e-s $d$ 'entrer dans les sphères commerciales des grandes entreprises multinationales éditoriales, siégeant généralement dans les pays occidentaux. II s'agit d'entrer dans la compétition entourant le jeu de la publication comme le souligne Chamayou (2009). À partir de ce constat, les scientifiques sont ujudged principally through their ability to write" (Bartkowski, Deem et Ellison, 2015, p.105), mais pas 
selon n'importes quels critères. Ce n'est pas une forme de libération des structures oppressives par l'écriture; au contraire, c'est un assujettissement aux forces de production. Dans cette écriture aliénante, plusieurs formes de connaissance sont réduites au silence et $n^{\prime}$ entrent pas dans le jeu de la publication du système qui suit les logiques d'un modèle capitaliste occidental et colonial (Lugones, 2008). Les chiffres sont probants: d'une part, et inégalement d'une région à l'autre du globe, l'écart entre les femmes et les hommes reste énormes en termes de citations (Maliniak, Powers \& Walter, 2013) et, d'autre part, la parole des femmes reste encore à «inventer» (Leclerc, 1974) puisque dans plusieurs pays, les femmes ne réussissent pas à occuper des postes de professeure au même titre que les hommes.

Probablement, dans le système néolibéral, l'écriture n'est plus aussi libératrice qu'elle l'était: elle nous insère plutôt dans un "fast-paced model» qui commence dès l'admission au doctorat ou, si possible avant pour se «démarquer des autres" (Bartkowski, Deem et Ellison, 2015). En plus, nous ne sommes que rarement lu-e-s, si accepté-e-s : «le peer review dépend toujours de la participation de spécialistes" (Titz, 2015) et "the total number of citations any one article receives over the course of its lifetime is smal/" (Maliniak, Powers et Walter, 2013, p.27). Suivant I'affirmation plutôt cynique de Chamayou (2009, p.217) en regard de la «surproduction, redondance infinie et saturation universelle d'articles dispensables" (qui n'est pas sans rappeler la logique consumériste de la uthrow away culture»), ne devrions-nous pas plutôt réorienter nos façons de diffuser la recherche?

Dans ce contexte, ces sont plutôt les intérêts du marché défendus par l'État, les organismes de subvention et les institutions internationales qui guident les chemins de la recherche scientifique. L'université n'apparaît donc plus comme un lieu dédié à la réflexion critique et à la résolution des problèmes sociaux. C'est plutôt une institution cooptée par la logique du marché et qui, comme le souligne Insel (2009), mènerait à une stérilisation de la science et à la production d'une société de la méconnaissance.

Le résultat de cette marchandisation du savoir est triple: premièrement, comme la pédagogie n'est pas très payante dans le monde néolibéral, I'enseignement devient une tâche secondaire que les professeure-s peuvent déléguer tout comme des gérants d'entreprise, deuxièmement, la culture de l'évaluation pousse vers des choix rationnels alignés selon les coûts-bénéfices (Chamayou, 2009); puis finalement, force est de constater une démotivation générale des étudiante-s qui se manifeste par des attitudes désengagées face à l'apprentissage, l'université s'imposant de plus en plus comme une "manufacture de diplômes" (Côté et Allahar, 2010, p.12).

\section{La pensée critique comme un outil de résistance}

Les nouvelles contraintes imposées par la néolibéralisation de l'université impose des nombreux défis à celles et ceux qui ont décidé de poursuivre des études aux cycles supérieurs. La logique de productivité accélérée et l'importance de répondre aux intérêts politiques et économiques, traduits dans les coupures budgétaires faites par l'état et dans les exigences des organismes de subvention des recherches et par les universités, nous forcent à mettre en veille nos intérêts de recherche pour jouer les règles qui y sont posées. Comme l'affirme Barkowski, Deem et Ellison, dans 
la logique de la productivité, le «mérite est défini en termes de la quantité et de qualité des publications" (2015, p.100). Ici, les conseils ironiques donnés aux enseignants-chercheurs par Chamayou (2009, p.213) sont étonnants. II affirme: "Oubliez la vérite. Cessez de vouloir changer la réalite. Coulez-vous dans le moule et mettez-vous sur les startingblocks». Ces mots sont véritablement bien répandus dans l'académie.

Dans ce contexte de productivité accélérée, de compression de financement étatique, d'accumulation de tâches administratives de travail des enseignante-s et d'élimination des programmes dans les universités (Mountz et al., 2015), il est pertinent de penser à la situation de celles et ceux qui ont décidé de poursuivre une carrière académique. II est possible de penser que la plupart des jeunes chercheur-e-s décident de suivre des études doctorales motivé-e-s par le désir d'approfondir leur réflexion critique, leur connaissance d'un sujet spécifique et/ou pour faire carrière comme chercheur-e et enseignant-e au sein d'une université. Alors, les effets de la néoliberalisation de l'institution sur leurs carrières n'est pas à négliger.

Cornut et Larivière (2012) nous présentent des données intéressantes à propos du profil des étudiant-e-s inscrit-e-s aux programmes de doctorat au Québec dans la période de 1997 à 2012. Selon ces informations, de façon générale, les doctorants-e- ont besoin de 7,2 ans pour conclure leurs études. D'entre eux, $19 \%$ reçoivent des financements du Conseil de recherches en sciences humaines (CRSH) ou du Fonds de Recherche du Québec Société et Culture (FORSC) - deux des principaux organismes de subvention de recherche. Aussi, ils affirment que le taux de placement des docteurs dans I'enseignement à l'université a atteint
$53 \%$ (soit dans un poste menant à la permanence soit comme chargé de cours ou au Collège d'enseignement général et professionnel - Cégep ${ }^{5}$ ). De plus, les auteurs soulignent que $46,4 \%$ des doctorants quittent leurs programmes avant d'obtenir le diplôme pour les raisons mentionnées dans la section précédente eu égard à la compétition accrue dans les institutions éducatives.

Ces chiffres nous permettent de comprendre qui sont les aspirant-e-s au doctorat. De même, ils sont source de préoccupation de la part des jeunes chercheur-e-s. II nous montre une durée presque deux fois plus longue que celle prévue pour finir les études doctorales, seulement un quatrième des étudiants reçoit des bourses d'études, un taux de décrochage surprenant de presque $50 \%$ des doctorants et aussi une incapacité de l'université d'accueillir les nouveaux docteurs. En fait, l'analyse des données nous permet de penser qu'elles ne sont que la concrétisation du nouveau visage de l'université néolibérale: théoriquement de libre accès à tous, mais qui rencontre son modèle de clivage et d'exclusion dans la réussite de la permanence.

Suivant cette logique, I'analyse des effets de la néolibéralisation de l'université nous amène à réfléchir aux possibles stratégies pour faire face à aux changements et aux conditions de productivité accélérée imposées par ce modèle. Une piste est que l'analyse des enjeux de "marchandisation du savoir" serait un outil indispensable dans la quête des solutions aux impositions du système universitaire actuel (Insel, 2009, p.142).

Si la pression du marché du savoir est celle de pousser les universitaires à une production exacerbée, la proposition de

5. Des institutions chargées des études pré-universitaires. 
"slow scholarship" faite par le collectif de féministes (Mountz et al., 2015, p.1238) nous semble intéressante. Selon les auteures, le mouvement du slow scholarship sıoppose à lraugmentation croissante des demandes de la vie académique et donc aux exigences de lsuniversité néolibérale. Dans le contrecourant des idéaux néolibéraux, ce cadre propose un engagement lent (nous pourrions dire, profond et réflexif) avec nos idées, nos objets de rechercher et nos champs d'action. Aussi, les auteures soulignent "the benefits of unexpected "disruptions" in the research and writing process" et donc limportance de Isamélioration de la qualité de lsécriture des recherches publiées. En bref, pour les auteures, le mouvement du slow scholarship est une stratégie de résistance aux présupposés néolibéraux dans le sens qu'il instaure un réseau de production collective, collaborative et qui propose des modèles d'action communautaires.

Les points soulevés nous incitent à critiquer les pratiques individualisées et à réorienter la façon de construire les milieux scientifiques à partir de stratégies collectives. Comme Denyse Baillargeon (1993, p.58) le fait remarquer, «il faut cesser de considérer que le changement social est l'unique résultante d'une action du public sur le privé, du structurel sur le personnel, et admettre la possibilité que l'inverse puisse aussi se produire ".

Une autre question pertinente concerne à l'employabilité des jeunes docteur-e-s. Les diverses facettes de la précarité de l'université, comme nous l'avons mentionné, ont poussé les discussions sur les fameux alt-ac, "alternative academic", (Bowess, 2015) qui offriraient des opportunités hors du champ purement scientifique pour satisfaire au problème découlant de l'augmentation des doctorant-e-s et de la diminution des postes disponibles (Bérubé, 2013; Grondin, D'Aoust et Racine-Sibulka, 2012). En ce sens, les activités en dehors des milieux scientifiques sont intéressantes pour le curriculum vitae des étudiant-e-s que pour l'acquisition de compétences professionnelles diversifiées. Ainsi, il s'agit de considérer que, d'un côté, l'expérience professionnelle hors université est un atout particulier pour la science en elle-même et, d'un autre côté, qu'il est important "de faire valoir à quel point certaines compétences - en matière de recherche, de pensée critique, d'enseignement ou de rédaction, par exemple -, sont prisées sur le marché du travail» (Bowness, 2015). Alors, former des doctorant-e-s ne doit pas avoir comme finalité unique la création d'emplois de professorat. Au contraire, la pensée critique qui est censée accompagner la réalisation d'un doctorat devrait avoir des effets tangibles sur la société en question. La recherche, comme extension de la curiosité humaine, ne devrait pas être limitée aux institutions universitaires.

Alors, la posture critique et engagée des chercheur-e-s, des enseignant-e-s et des étudiant-e-s est au coeur des stratégies de résistance et de remise en question de l'université néolibéral et de son rôle social. Elle doit d'une part, composer avec les aléas du néolibéralisme et ses impressionnants impacts sur la pensée (Giroux, 2015) et elle doit aussi contester les conditions de sa propre formation. S'engager sur ce terrain est un défi de taille. Cela implique une remise en question des politiques "désincorporées" issues des Lumières (Callon, 1999, p.73) et la construction d'une épistémologie critique qui pose un regard sur sa propre édification (Jasanoff, 1996). Être un-e intellectuel-le engagé-e signifie aussi adhérer à certains processus d'autoréflexion sur la pratique scientifique 
et pédagogique. C'est en ce sens que nous devons constamment interroger "the ways in which our scholarly work may play out in the arenas of the 'real world'» (Jasanoff, 1996, p.409).

La possibilité de rendre visible les inégalités sociales, les rapports de forces ou les effets néfastes du néolibéralisme sont ainsi multiples et, à notre avis, cela est au centre du rôle social des universités. Dans ce contexte, comme I'affirme Giroux (2015), I'université est probablement l'un des derniers bastions qui permette de poser un regard critique sur nos démocraties, ouvrant des espaces publics de débats et confrontant le modèle néolibéral d'organisation du social. C'est I'université qui permet de trouver des outils théoriques et méthodologiques pour construire des narratives collectives (Giroux, 2015, p.181) et faire le contrepoids aux discours homogénéisants. Ainsi, le rôle des intellectuel-les est peut être celui de porte-parole comme le signifie Callon (1999) en ce sens qu'il permet de rendre visible des réalités qui ne le seraient pas sans la médiation de divers acteurs et actrices permettant son intelligibilité. II s'agit donc d'utiliser les outils que nous créons pour amener le dissensus - par opposition au consensus - sur les questions sociales : c'est ce qui permettrait même de revitaliser la démocratie comme I'argumente Judith Butler (2015).

Finalement, la résistance au modèle d'université néolibérale se fait surtout dans la remise en questions des impositions qui bénéficient, privilégient et produisent des savoir hégémoniques - et, donc, blancs, masculins et occidentaux. Dans ce contexte, comme l'argumente Muller, nos plus grands défis est celui de soulever des questions critiques dans les contextes de "uncritical thinking". Pourtant, la stratégie proposée par lıauteur nous apparaît pertinente. II affirme:

"[...] sowing the seeds of doubt, raising critical questions, and highlighting the power embedded in the genre of expert testimony, one can maintain a commitment to the critical enterprise, and bring the experience back to one's own research agenda, and thus engage more critically in academic forums in ways only made possible through one's own participation» (2013, p.12).

Soulever des critiques et possibilités de résistance est le but ultime de cet article: la section suivante cherche à réfléchir aux opportunités de contestation des savoirs hégémoniques à l'intérieur du système néolibéral.

\section{L'université néolibérale et la contestation des savoirs hégémoniques}

Les réflexions présentées nous amènent à réaffirmer que la décision d'entreprendre un doctorat dans une langue autre que l'anglais est lourde en conséquences comme l'exposent Grondin, D'Aoust et Racine-Sibulka (2012). Comme nous l'avons démontré, une des caractéristiques de l'université néolibérale est qu'elle perpétue les privilèges et marque une continuité avec les impératifs de la société capitaliste. Par exemple, comme la langue anglaise s'est imposée du point de vue commercial, elle donne aussi le ton pour les publications scientifiques, marginalisant les autres langues dans l'économie du savoir. La langue d'écriture devient donc à la fois une barrière à la diffusion des savoirs, mais aussi un autre facteur de stress pour les doctorant-e-s désirant promouvoir leurs recherches. Selon nous, il s'agit d'un exemple de plus montrant comment le 
modèle universitaire néolibéral contribue au renforcement du savoir hégémonique qui met à mal la diversité des formes de pensée.

L'exemple de l'hégémonie de la langue anglaise au sein de la communication scientifique nous donne des pistes pour remettre en question les savoirs hégémoniques en question. Qu'en estil des millions de personnes dont la langue maternelle n'est pas l'anglais? Ces personnes ne bénéficient pas du statut de "producteur» ou "productrices» du savoir; leur visibilité est réduite dans le système-monde et les structures impériales découlant de l'épistémologie moderne contribuent à maintenir les inégalités dans la production de la connaissance. Comme Mignolo et Tlostanova l'argumentent (2009), I'anglais agit comme une langue impériale pour produire des savoirs impériaux, imposant une pensée hégémonique occidentale.

Il est donc possible donc d'affirmer que le capitalisme cognitif a amené une construction du savoir et des pratiques épistémologiques violentes qui ont rendu invisibles les savoirs "autres" et "subalternes». Cette colonisation du savoir est ce que Sousa Santos appelle "l'épistémicide» et qui se caractérise par la suppression des savoirs non-occidentaux (2010, p.68). L'épistémologie moderne s'est construite à partir de la notion "d'égo-politique de la connaissance": c'est-à-dire, sur la prémisse qu'il existe une façon de produire de la connaissance qui serait non située, déhistoricisée et décorporalisée (Grosfoguel, 2006; Restrepo et Rojas, 2010), C'est sur ces bases que s'est construite l'université néolibérale telle que nous la connaissons aujourd'hui.

De ce constat, il apparaît que l'université néolibérale a bien réussi son travail: les scientifiques ne questionnent pas cette incapacité de l'université à sortir des cadres de production de la connaissance ordonnés par la modernité politique et qui sous-tendent des inégalités politiques et sociales. C'est de cette façon que I'université perpétue plusieurs systèmes d'oppression comme le patriarcat: l'université néolibérale a des «embodied effects" (Mountz et al., p. 1238) visibles notamment par les hiérarchies de genre qui subsistent dans les départements et facultés. De fait, les effets du "new managerialism" se font davantage ressentir selon le genre McRobie (2015), ayant par exemple un plus grand impact sur les femmes par exemple. Ainsi, il est possible d'identifier une problématique d'intersection des oppressions dans les universités contemporaines qui représente l'aboutissement de la matrice coloniale de domination, soit l'intégration des schèmes de dominations par les dominé-e-s. C'est cette matrice qui nous fait voir le monde scientifique nordaméricain et européen (nous pourrions dire occidental, masculin et blanc) comme étant l'idéal de productivité à atteindre. Dans cette logique marchande, la production subalterne, à partir des marges ou décoloniale est perçue à partir du cadre de cette matrice coloniale: ces savoirs sont conçus alors comme s'ils présentaient un "retard" épistémique sur I'Occident. C'est ainsi que Data Gueule montre que la plupart des pays de la périphérie selon la conceptualisation de Dussel, n'ont pas accès aux publications scientifiques des grands empires de l'édition (Data Gueule, 2016). C'est une racisation du savoir que l'on refuse de voir.

L'université néolibérale cache alors un système d'homogénéisation de la pensée qui contribue à la construction des savoirs hégémoniques. C'est la logique de productivité, la structure de pensée occidentale qui dictent les normes de 
production du savoir scientifique. Or, si I'université est l'endroit de construction de la connaissance, de la formation à la pensée critique, elle devrait être aussi un lieu de respect et de valorisation de la diversité. En ce sens, la contestation de l'hégémonie du savoir passe nécessairement par une "désobédience épistémique» comme le souligne Mignolo qui permettrait de remettre en question les bases du savoir: "de-linking implies epistemic disobedience rather than the constant search for "newness"" (Mignolo, 2011, p.45). Ainsi, au lieu de concevoir l'université comme un site d'accumulation des connaissances, il $s^{\prime}$ agit de considérer et co-construire des épistémologies alternatives à celles dérivées de la méthode cartésienne dans le but d'influer sur les rapports de pouvoir existants qui sont souvent perpétués par les institutions universitaires à différents degrés dans le système-monde.

\section{Conclusions}

Est-ce que I'université est encore le lieu de construction critique de la connaissance? Est-ce toujours un endroit de problématisation, de réflexions et de discussions enrichissantes? Est-ce que I'université contribue à donner des solutions théoriques et pratiques aux problèmes sociaux? II semble que I'université néolibérale ait mis à mal ces piliers de la construction de la connaissance. Les intellectuel-le-s semblent saturé-e-s par des contraintes institutionnelles et économiques qui leur échappe et peinent à penser à l'extérieur de ces structures oppressantes. Comme nous l'avons soulevé dans ce texte, la fonction marchande de la recherche pousse les professeur-e-s à toujours devoir se surpasser en termes de nouveautés pour répondre aux demandes du capitalisme cognitif (Koromyslova et Depelteau, 2015).
Dans cet article, notre objectif était de dresser les contours de I'université néolibérale et d'évaluer ses transformations dans le temps afin de réfléchir sur les possibilités $d^{\prime} y$ résister. Nous avons démontré que la gouvernance par les chiffres, le modèle de gestion par objectifs et toutes les autres façons d'encourager la compétitivité et la concurrence sont une des valeurs soulevées par les différent-e-s auteur-e-s analysé-e-s comme des caractéristiques de l'économie du savoir.

Au contraire, pour nous comme pour Insel, "la connaissance est intrinsèquement un bien collectif» (2009, p.151) et ne devrait pas être sujette aux aléas de l'économie capitaliste néolibérale. Nous nous considérons comme des scientifiques, féministes et engagées qui désirent transformer les réalités sociales: suivant Foucault (cité par Sapiro, 2009), les scientifiques engagé-e-s doivent servir de pont entre les différentes sphères sociales pour permettre une prise de conscience collective. Comme I'affirme Sotello Viernes Turner, "by bringing ourselves through the door and supporting others in doing so as well, we can define ourselves in and claim unambiguous empowerment, creating discourses that address our realities, affirm our intellectual contributions, and seriously examine our worlds" (2002, p. 89).

Pour nous, agir sur les méfaits de I'université néolibérale c'est combattre les structures rigides des rapports entre savoir et pouvoir. Pour se faire, il faut agir sur les fissures comme le soutient Foucault (2009). La démocratisation de l'accès aux cycles supérieurs basée sur des discours méritocratiques qui cachent les inégalités de conditions d'études à qui sont soumis les étudiants; les conditionnalités imposées par des organismes de 
subvention; I'anglais comme langue universelle; la publication dans les revues internationales renommées comme le critère major d'évaluation d'un chercheur, tous ces points de faiblesses doivent être contestés.

Le travail de résistance contre ces structures néolibérales est ardu, mais possible: I'université n'est pas appelée à disparaître; selon nous, c'est l'université elle-même qu'il faut subvertir et c'est à partir de cette position que nous pourrons réellement changer la donne. L'université est une institution sociale essentielle qui, en dépit de ses difficultés à s'auto-analyser (Oliver, 2015), a toujours une possibilité de s'auto-transformer et de transformer la réalité sociale dans laquelle elle évolue. Nous sommes d'avis que les chercheur-e-s et que l'université comme institution ont comme fonction première la transformation sociale et nous voyons dans la pensée critique sur les valeurs et les pratiques qui guident les actions de l'université néolibérale un outil indispensable de subversion de ce système. Alors, c'est dans ce contexte que la remise en question des savoirs hégémoniques, la remise en doute des demandes et des exigences de productivité, la réflexion sur le rôle social de l'université et les actions comme celle du mouvement de slow scholarship nous apparaissent comme des outils importants à la résistance aux impositions néolibérales et, principalement, dans la construction d'un modèle d'université autonome dont la quête de savoir est motivée par la curiosité intellectuelle et par l'envie de proposer des solutions aux problèmes politiques, économiques et sociaux.

\section{Références}

Baillargeon, D. (1993). Histoire orale et histoire des femmes : itinéraires et points de rencontre. Recherches féministes, 6(1), 53-68.

Bartkowski, J.P, Deem, C.S. et Ellison, C.G. (2015). Publishing in Academic Journals: Strategic Advice for Doctoral Students and Academic Mentors. The American Sociologist, 46(1), 99-115.

Berubé, M. (2013). The Humanities, Unraveled. The Chronicle Review, 18, Récupéré le 23 juin 2017 de: http://chronicle.com/article/HumanitiesUnraveled/137291/

Bourdieu, P. (1979). La Distinction. Critique sociale du jugement. Paris : Les Editions de Minuit.

Bowess, S. (2015). Sensibilisation aux carrières non universitaires. Affaires universitaires. Récupéré le 23 juin 2017 de http://www.affairesuniversitaires. $\mathrm{ca} /$ articles-de-fond/article/sensibilisation-auxcarrieres-non-universitaires/

Butler, J. (2015). Cuerpos que todavía importan. Canal Youtube d'UNITREF. Récupéré le 23 juin 2017 de https://www.youtube.com/watch?v=-UP5xHhz17s

Callon, M. (1999). Ni intellectuel engagé, ni intellectuel dégagé : la double stratégie de l'attachement et du détachement. Sociologie du travail, 41(1), 65-78.

Chamayou, G. (2009). Petits conseils aux enseignantschercheurs qui voudront réussir leur évaluation. Revue du Mauss, 1(33), 208-226.

Clavier, C. et D'Aoust, A.-M. (2017). Plan de cours POL9020 Séminaire de préparation à la rédaction de textes scientifiques et aux activités de recherche. Université du Québec à Montréal.

Considine, M. (2006). Theorizing the University as a Cultural System: Distinctions, Identities, Emergencies. Educational Theories, 56(3), 255-270.

Cornut, J. et Larivière, V. (2012). Docteurs et doctorants en science politique au Québec (1997-2010). Politique et Sociétés, 31(3), 67-86.

Côte, J. et Allahar, A.L. (2010). Tout n'est pas si rose au paradis. Dans J.E. Côté (dir.), La tour de papier: l'universite, mais à quel prix?' (p. 33-82). Montréal : Éditions Logiques.

Data Gueule. (2016, 17 octobre). Privés de savoir? Data Gueule \#63. Récupére le 23 juin 2017 de : https://www.youtube.com/watch?v=WnxqoPc0ZE\&feature =youtu.be

De Sousa Santos, B. (2010). Para descolonizar Occidente: más allá del pensamiento abismal. Buenos Aires: CLACSO.

Foucault, M. (2009). Nietzsche, a Genealogia e a História. Dans M. Foucault, Microfísica do poder (pp.15-38). São Paulo: Graal.

Gingras, Y. (2004). L'universite en mouvement. Égalite: Revue de la Sociéte acadienne dranalyse politique, $50,13-28$.

Giroux, H.A. (2015). The Retreat of Academics as Public Intellectuals. Les Cahiers de l'idiotie, 6, 159-181.

Grondin, D., D'Aoust, A.-M. et Racine-Sibulka, P. (2012). La discipline des Relations internationales au Québec et au Canada : pistes de réflexions sur les enjeux et dilemmes linguistiques liés au marché du travail. Politique et Sociétés, 31(3), 9-37.

Grosfoguel, R. (2006). La descolonización de la economía política y los estudios postcoloniales: transmodernidad, pensamiento fronterizo y colonialidad global. Tabula Rasa, 4(1), 17-46.

Insel, A. (2009). Publish or Perish! La soumission formelle de la connaissance au capital. Revue du Mauss, $1(33), 141-153$. 
Jasanoff, S. (1996). Beyond Epistemology: Relativism and Engagement in the Politics of Science. Social Studies of Science, 26(2), 393-418.

Koromyslovae, N. et Depelteau, J. (2015). Marche ou crève : économie de la subsistance aux cycles supérieurs. Les Cahiers de l'idiotie, 6, 263-271.

Leclerc, A. (1974). Parole de femme. Paris : Éditions Grasset \& Fasquelle.

Lesemann, F. (2003). La société des savoirs et la gouvernance : la transformation des conditions de production de la recherche universitaire. Lien social et politiques, 50, 17-37.

Lugones, M. (2008). The coloniality of gender. Worlds \& Knowledges Otherwise, 2, 1-17.

Maliniak, D., Powers, R. et Walter, B.F. (2013). The Gender Citation Gap in International Relations, International Organization, 67(4), 889-922.

McRobie, A. (3 septembre 2015). Women's Working Lives in the Managerial University and the Pernicious Effects of the "Normal" Academic Career. London School of Economics. Récupéré le 23 juin 2017 de: http://blogs.Ise.ac.uk/ impactofsocialsciences/2015/09/03/womensworking-lives-in-the-managerial-university/

Meulemeester, J.-L. (2011). Quels modèles d'universite pour quel type de motivation desacteurs? Une vue évolutionniste. Pyramides: revue du centre d'études et de recherches en administration publique, 21, 261-289.

Mignolo, W. (2011). Epistemic Disobedience and the Decolonial Option: A Manifesto. Transmodernity: Journal of Peripheral Cultural Production of the Luso-Hispanic World, 1(2), p.45.

Mignolo, W. et Tlostanova, M. (2009). Habitar los dos lados de la frontera/teorizar en el cuerpo de esa experiencia. Revista IXCHEL, 1, pp.1-22. Récupéré le 23 juin 2017 de: http://www.redkatatay.org/sitio/ talleres/mignolo_frontera.pdf
Mountz et al., A. (2015). For Slow Scholarship: A Feminist Politics of Resitance through Collective Action in the Neoliberal University. ACME: International E-Journal for Critical Geographies, 14(4), 12081234.

Muller, B. (2013). Testifying While Critical: Notes on Being an Effective Gadfly. Dans M.B. Salter et Mutlu, C.E. (dir.), Research Methods: In Critical Security Studies (pp.109-119). Londres et New York: Routledge.

Ó Loingsigh. G. (2016). Experiencias internacionales de paz: mitos y realidades. Conférence presenté au XIV Congreso Internacional de Humanidades: Escenarios posible en el posconflicto colombiano. Bucaramanga, Colombie.

Olivier, L. (2015). Université: du sacré au profane. Les cahiers de l'idiotie, 6, 331-344.

Paterson, M. (2015). The University, the Industrial Machine, the 'Critical' Professor. Les Cahiers de l'idiotie, no. 6, 221-231.

Sapiro, G. (2009). Modèles d'intervention politique des intellectuels : le cas francais. Actes de la recherche en sciences sociales, 1 (176-177), 8-31.

Sotello Viernes Turner, C. (2002). Women of Color in Academe: Living with Multiple Marginality. The Journal of Higher Education, 73(1), 74-93.

Restrepo, E. et Rojas, A. (2010). Inflexión decolonial: fuentes, conceptos y cuestionamientos. Popayán: Editorial Universidad del Cauca.

Titz, S. (2015). Un peer-review à revoir. Horizons, 106, 13-17.

Tupper, A. Pocklington, T.C. (2002). No Place to Learn: Why Universities Aren't Working, Vancouver: UBC Press. 